\title{
FATORES DE RISCO MATERNOS PARA PREMATURIDADE EM UMA MATERNIDADE PÚBLICA DE IMPERATRIZ-MA
}

\author{
Adriana Carvalho de ALMEIDA ${ }^{\mathrm{a}}$, Ana Cristina Pereira de JESUS ${ }^{\mathrm{b}}$, Pamylla Fortes Tanikawa LIMAc, \\ Márcio Flávio Moura de ARAÚJO ${ }^{\mathrm{d}}$, Thiago Moura de ARAÚJO
}

\section{RESUMO}

Apesar dos avanços da obstetrícia, a prematuridade ainda se configura como um dos grandes problemas de saúde pública, em virtude da morbidade e da mortalidade neonatal que causa. O objetivo deste estudo foi investigar fatores de risco maternos para nascimentos de prematuros em uma maternidade pública de Imperatriz-MA. Um estudo comparativo transversal foi realizado com 116 puérperas, por meio de entrevistas estruturadas. Os dados coletados foram analisados através do Software Epi-Info, versão 3.5.1. As variáveis que tiveram associação estatisticamente significante com a prematuridade foram: renda mensal inferior a 2 salários mínimos $(p=0,046)$; estresse na gestação ( $p=0,027)$; primiparidade ( $p=0,044)$; assistência pré-natal ausente ou inadequada $(p<0,001)$; e intercorrências clínicas na gestação $(\mathrm{p}=0,001)$. Os resultados apontam que os fatores de risco maternos implicados na prematuridade estão relacionados, sobretudo, com hábitos de vida, assistência pré-natal e condições socioeconômicas e clínicas.

Descritores: Fatores de risco. Trabalho de parto prematuro. Saúde da mulher.

\section{RESUMEN}

A pesar de los avances en la obstetricia y la prematuridad sigue siendo un problema importante de salud pública debido a la morbilidady mortalidad neonatal que causa. El objetivo de este estudio fue investigar los factores de riesgo maternos para los nacimientos prematuros en una maternidad pública en Imperatriz, MA. Un estudio comparativo de la sección transversal se llevó a cabo con 116 madres, a través de entrevistas estructuradas. Estudio caso-control se realizó con 116 mujeres, agrupadas en el caso de (58) y control (58), a través de entrevistas estructuradas. Los datos fueron analizados con Epi-Info versión 3.5.1. Las variables que mostraron una asociación estadísticamente significativa con el parto prematuro fueron ingresos mensuales $<2$ salarios mínimos ( $p=0,046)$, el estrés durante el embara $\approx 0$ ( $p=0,027)$, primiparidad $(p=0,044)$, atención prenatal ausente o inadecuada $(p<0,001)$ y complicaciones clínico durante el embarazo $(p=0,001)$. Los resultados muestran que los factores de riesgo implicados en la prematuridad materna están relacionadas principalmente con el estilo de vida, factores socioeconómico y clínico y la atención prenatal.

Descriptores: Factores de riesgo. Trabajo de parto prematuro. Salud de la mujer.

Título: Los factores maternos de riesgo de parto prematuro en una maternidad pública de Imperatriz-MA.

\section{ABSTRACT}

Despite advances in obstetrics, prematurity is still a major public health problem because of the neonatal morbidity and mortality it causes. The objective of this study was to investigate maternal risk factors for premature births in a public maternity in the city of Imperatriz-MA. A cross-sectional comparative study was conducted with 116 mothers, through structured interviews. The data were analyzed using Epi-Info version 3.5.1. The variables that showed statistically significant association with preterm delivery were monthly income below two minimum wages $(p=0.046)$, stress during pregnancy $(p=0.027)$, primiparity $(p=0.044)$, absent or inadequate prenatal care $(p<0.001)$ and clinical complications in pregnancy $(p<0.001)$. The results show that the maternal risk factors implicated in prematurity are related mainly with lifestyle, socioeconomic and clinical variables, and prenatal care.

Descriptors: Risk factors. Premature labor. Women's health.

Title: Maternal risk factors for premature births in a public maternity hospital in Imperatriz-MA.

a Enfermeira. Enfermeira do Centro Oncológico Brasileiro - COBRA, Imperatriz, Maranhão, Brasil.

b Enfermeira Especialista em Enfermagem Neonatal e Obstétrica. Mestranda do Programa de Pós-Graduação em Enfermagem da Universidade

Federal do Ceará (UFC). Professora do Curso de Enfermagem da Universidade Federal do Maranhão (UFMA). Imperatriz, Maranhão, Brasil.

c Terapeuta Ocupacional. Terapeuta Ocupacional do Hospital Regional de Imperatriz. Imperatriz, Maranhão, Brasil.

d Doutor em Enfermagem. Professor do Curso de Enfermagem da UFMA. Imperatriz, Maranhão, Brasil.

e Mestre em Enfermagem. Doutorando do Programa de Pós-Graduação em Enfermagem da UFC. Professor do Curso de Enfermagem da UFMA. Imperatriz, Maranhão, Brasil. 


\section{INTRODUÇÃO}

O parto prematuro é definido como a ocorrência do nascimento antes do termo, ou seja, crianças nascidas antes da maturidade fetal. A característica mais utilizada para classificá-lo é de ordem cronológica: nascimento antes de 37 semanas de gestação, contadas a partir do primeiro dia do último período menstrual ${ }^{(1)}$.

O nascimento de prematuros representa um grande desafio para os serviços de saúde pública em todo o mundo, por tratar-se de um determinante de morbi mortalidade neonatal. No mundo, atualmente, a prematuridade representa a principal causa de mortalidade neonatal com um percentual de $75 \%^{(2,3)}$. As prevalências de prematuridade no mundo, no Brasil e no Maranhão são de 9,2\%; 7,1\%; e 17,3\%, respectivamente ${ }^{(4,5)}$. Além das repercussões clínicas, existem ainda as implicações econômicas associadas a esse evento, uma vez que demanda cuidados de maior complexidade que devem ser tomados mesmo depois do período neonatal, muitas vezes por toda a vida, devido a sequelas causadas pela prematuridade $^{(6)}$.

Apesar dos avanços na obstetrícia, o número de nascimentos de prematuros ainda é elevado, principalmente em países subdesenvolvidos e em desenvolvimento, como o Brasil, onde se observa uma tendência de aumento dos $\operatorname{casos}^{(7,8)}$. Dos diversos fatores de risco maternos que estão implicados na prematuridade, o levantamento bibliográfico desta pesquisa permite destacar os seguintes como os mais prevalentes e importantes: os hábitos de vida, as condições socioeconômicas, os antecedentes ginecológicos e obstétricos, as intercorrências gestacionais e a assistência pré-natal ausente ou inadequada ${ }^{(1,9)}$.

Algumas pesquisas consultadas constataram que a ocorrência de alguns fatores sociodemográficos e clínicos, associados à prematuridade, é mais prevalente e importante que a de outros. Por exemplo, no que diz respeito aos fatores sociodemográficos, a idade $\leq 18$ e $\geq 35$ anos, o baixo peso pré-gestacional, o uso de substâncias como tabaco e álcool, o baixo nível socioeconômico, ser solteira, possuir baixa escolaridade e o estresse na gestação são determinantes para um desfecho gestacional negativo. Por sua vez, os dados clínicos maternos preponderantes na gestação são: possuir recém-nascido prematuro (RNPT) prévio; apresentar intercorrências na gestação (doença hipertensiva específica da gestação DHEG, sangramentos vaginais e infecção do trato urinário - ITU, dentre outras); e ausência de cuidados pré-natais ${ }^{(1,9-11)}$.

No município de Imperatriz-MA, o segundo maior do Estado do Maranhão e importante polo de saúde para os Estados do Maranhão, Tocantins e Pará, inexistem dados epidemiológicos referentes à temática abordada, conforme consulta em bases de dados digitais e da própria Secretaria de Saúde do município em questão. Dessa forma, o estudo teve como objetivo investigar os fatores de risco maternos associados ao nascimento de prematuros em uma maternidade pública de Imperatriz-MA. Certamente, o presente estudo poderá contribuir para o conhecimento da temática da saúde perinatal e para a elaboração de políticas públicas voltadas para a prevenção dos fatores de risco maternos à prematuridade nesta região.

\section{MÉTODO}

Trata-se de um estudo quantitativo com delineamento transversal, desenvolvido em uma maternidade pública de médio porte, referência para a assistência obstétrica e neonatal, localizada no município de Imperatriz-MA, responsável pelo atendimento de cidades do Sul do Maranhão, Pará e Tocantins.

$\mathrm{Na}$ ocasião do estudo, no segundo semestre de 2010,com base em informações da unidade de vigilância epidemiológica da maternidade em questão, foram realizados 497 partos, sendo $20 \%$ deles de prematuros $(\mathrm{N}=99)$. Dessa forma, esta pesquisa contou com uma amostra conveniente de 116 puérperas, o que equivale a $23 \%$ da população em estudo, agrupadas em dois grupos. No primeiro (grupo A), foram incluídas 58 puérperas que tiveram seus bebês prematuros $(<37$ semanas de gestação) e estavam internadas. Por sua vez, no segundo (grupo B),foram incluídas 58 puérperas de partos a termo ( $\geq 37$ semanas de gestação) que se encontravam internadas nas enfermarias das alas das enfermarias ou nos alojamentos da instituição em questão. Foram entrevistadas as puérperas que concordaram em fazer parte de todo o seguimento da pesquisa e que se encaixavam no critério de elegibilidade para a amostra, a saber: ser puérpera de feto único e vivo. Foram excluídas da pesquisa todas as puérperas cujo parto pré-termo tivesse sido induzido por complicações do feto. 
A coleta de dados ocorreu no período de novembro de 2010 a janeiro de 2011 , por meio de entrevistas para aplicação de um questionário estruturado contemplando as seguintes variáveis: fatores socioeconômicos (idade, cor, escolaridade, estado civil, ocupação, renda familiar mensal, estresse); fatores clínicos maternos durante a gestação (tabagismo, álcool, índice de massa corporal-IMC, história obstétrica, intercorrências na gestação recente, número de consultas pré-natais e trimestre gestacional da primeira consulta pré-natal).

Vale destacar para o quesito renda familiar, que o valor do salário mínimo (SM) no período do estudo foi de $\mathrm{R} \$ 545,00$ reais. No caso do IMC, levou-se em conta o peso e a altura anteriores à gestação das puérperas registrados nas fichas de pré-natal, pois como as avaliadas eram puérperas, a tomada do IMC atual seria um viés metodológico. A avaliação do estresse foi subjetiva, uma vez que não se utilizou nenhum instrumento psicométrico, levando-se em conta apenas as informações fornecidas pelas puérperas.

Os aspectos éticos para a execução da pesquisa foram respeitados, de maneira que antes de iniciar as entrevistas com as puérperas foi realizada a explicação dos objetivos da pesquisa e dada a garantia deque as informações fornecidas seriam mantidas em sigilo e utilizadas apenas para fins científicos. Depois de oferecidas essas informações, todas as entrevistadas decidiram participar do estudo e assinaram o Termo de Consentimento Livre e Esclarecido (TCLE). Como princípio ético e global, o projeto de pesquisa foi submetido ao Comitê de Ética e Pesquisa (CEP) do Hospital Universitário da Universidade Federal do Maranhão (HUUFMA), tendo sido aprovado sob registro 004546/2010-10, conforme as recomendações previstas pela Resolução 196/96 do Conselho Nacional de Saúde (CNS).

Os dados coletados foram inicialmente digitados em planilha eletrônica do programa Excel versão 2007 e, em seguida, analisados pelo Software Epiinfo, versão 3.5.1. Foram calculadas as $O d d s$ Ratio (OR) (razões de chance) e consideradas significativas as que apresentaram $\mathrm{OR}>1$ e o intervalo de confiança adotado foi de $95 \%$. Foi utilizado ainda o teste do Qui-Quadrado para análise da associação das variáveis investigadas nos grupos A e B. Em todos os cruzamentos, foi adotado um nível de significância de $5 \%(\mathrm{p}<0,05)$.

\section{RESULTADOS E DISCUSSÃO}

A amostra do presente estudo foi composta de 116 puérperas, agrupadas em dois grupos, com e sem prematuridade. Essa amostra foi adequada para as análises quantitativas deste manuscrito, visto que alguns estudos semelhantes evidenciaram diferenças estatisticamente significantes com amostras que variaram de 58 a 579 nascimentos prematuros ${ }^{(3,12)}$.

De acordo com os resultados apresentados neste artigo, as variáveis que tiveram associação estatisticamente significante com a prematuridade foram: renda mensal $<2 \mathrm{SM}(\mathrm{OR}=2,67)$ e estresse na gestação $(\mathrm{OR}=2,41)$. Gestantes com renda inferior a dois SM ou que vivenciaram durante a gestação situações estressantes apresentaram duas vezes mais chances de terem seus bebês prematuros (Tabela 1).

Apesar desta pesquisa não ter detectado diferenças estatisticamente significantes entre os grupos A e B no pareamento com a idade ( $p=0,561)$,alguns autores têm relacionado os extremos da vida reprodutiva com a prematuridade. Em estudo realizado no Estado de Santa Catarina, a prematuridade esteve associada às mães com idade $<20$ anos e $>40$ anos ${ }^{(2)}$. Outra publicação consultada averiguou que a maior frequência de prematuridade entre adolescentes não ocorre apenas em virtude do ponto de vista obstétrico, mas principalmente pelas consequências psicológicas e socioeconômicas que uma gravidez na adolescência pode acarretar ${ }^{(13)}$.

Outra constatação deste estudo foi o fato de que $60 \%$ das mães que tiveram seus filhos prematuros não tinham uma atividade profissional fora do lar. Como para este quesito os grupos A e B apresentaram o valor absoluto, não foi possível detectar diferenças estatisticamente significantes na associação entre atividade profissional e prematuridade $(\mathrm{p}=0,345)$ (Tabela 1$)$.

Nesta investigação, mais de $70 \%$ das mulheres de ambos os grupos se declararam pardas, assim, para fins estatísticos, agrupou-se a amostra em puérperas pardas e não pardas. No entanto, mesmo com o predomínio das mulheres pardas, a maior incidência de prematuros nessa cor não obteve relevância estatisticamente significante nesta pesquisa $(\mathrm{p}=0,672)$. Alguns estudos executados no Brasil têm apontado dificuldades na mensuração da cor dos pesquisados, dada a característica multirracial de nossa sociedade, fato que talvez possa também ter dificultado o cruzamento dessas informações ${ }^{(14,15)}$. Segundo autores 
Tabela 1 - Distribuição das variáveis socioeconômicas das mães de crianças prematuras e a termo. Imperatriz, MA, 2011.

\begin{tabular}{llllllll}
\hline \multicolumn{1}{l}{ Prematuro } & \multicolumn{2}{l}{ Não Prematuro } \\
\hline VARIÁVEIS & $\mathrm{N}$ & $\%$ & $\mathrm{~N}$ & $\%$ & OR & IC & $\mathrm{p}$
\end{tabular}

Idade

$\begin{array}{llllllll}<20 \text { ou } \geq 35 \text { anos } & 23 & 40 \% & 23 & 40 \% & 1,24 & 0,58-2,65 & 0,561 \\ 20 \text { a } 34 \text { anos } & 35 & 60 \% & 35 & 60 \% & & & \end{array}$

Cor

Parda

Não parda

$43-74 \%$

$\begin{array}{llll}15 & 26 \% & 16 & 28 \%\end{array}$

Escolaridade (anos)

$\begin{array}{llllllll}<8 \text { anos } & 25 & 43 \% & 29 & 50 \% & 0,75 & 0,36-1,57 & 0,453 \\ \geq 8 \text { anos } & 33 & 57 \% & 29 & 50 \% & & & \end{array}$

Estado civil

$\begin{array}{llllllll}\text { Solteira } & 13 & 22 \% & 9 & 16 \% & 1,57 & 0,61-4,03 & 0,344 \\ \text { Casada/rel. estável } & 45 & 78 \% & 49 & 84 \% & & & \end{array}$

Ocupação

\begin{tabular}{|c|c|c|c|c|c|c|c|}
\hline Do lar & 35 & $60 \%$ & 35 & $60 \%$ & 0,7 & $0,33-1,47$ & 0,345 \\
\hline Atividade laboral & 23 & $40 \%$ & 23 & $40 \%$ & & & \\
\hline \multicolumn{8}{|l|}{ Renda Mensal } \\
\hline$<2 \mathrm{SM}$ & 47 & $89 \%$ & 41 & $75 \%$ & 2,67 & $0,94-7,59$ & $0,046^{*}$ \\
\hline$\geq 2 \mathrm{SM}$ & 6 & $11 \%$ & 14 & $25 \%$ & & & \\
\hline \multicolumn{8}{|l|}{ Estresse } \\
\hline Sim & 41 & $71 \%$ & 29 & $50 \%$ & 2,41 & $1,12-5,18$ & $0,027 *$ \\
\hline Não & 17 & $29 \%$ & 29 & $50 \%$ & & & \\
\hline
\end{tabular}

britânicos, ser mulher de cor negra é fator predisponente ao nascimento de prematuros ${ }^{(16)}$.

No que diz respeito à associação entre escolaridade e prematuridade, não foram evidenciadas divergências estatisticamente significantes entre os percentuais dos grupos A e B desta investigação $(p=0,453)$. Tal achado foi similar ao encontrado em outras pesquisas desenvolvidas no Maranhão e em Santa Catarina ${ }^{(2,17)}$. Por sua vez, foi identificada uma investigação que concluiu que quanto maior for a quantidade de anos de estudo apresentados pela futura mãe menor será o risco de um parto prematuro $^{(18)}$.

Embora o número de solteiras entre as mães de prematuros tenha sido maior do que o de mães a termo, não foi possível constatar evidência estatisticamente significante nesse cruzamento $(\mathrm{p}=0,342)$. Outra pesquisa consultada detectou resultado diferente, apontando as solteiras como mais vulneráveis ao nascimento de bebês prematuros ${ }^{(19)}$. Talvez isso ocorra em virtude da falta de um companheiro para dividir ou compartilhar as dificuldades e responsabi- 
lidades dessa fase, o que pode levar a uma gestação mais estressante, desencadeando um parto antes do tempo previsto(10).

Quanto à renda mensal, $89 \%$ das mães de prematuros possuíam renda familiar mensal de menos de 2 salários mínimos, evidenciando um dado com significância estatística $(\mathrm{p}=0,046)$ nesta associação, corroborando resultados de estudos semelhantes realizados no Sul do Brasil ${ }^{(8,17)}$. O baixo nível socioeconômico tem sido relatado na literatura como um fator de risco importante para nascimentos prematuros, o que pode ser explicado pela associação com outros fatores predisponentes para tal evento, tais como, nutrição deficiente, trabalho excessivo, maior estresse físico e psicológico, assistência em saúde inadequada na gestação, etc. Isolados ou em conjunto, esses fatores aumentam as chance de um parto antecipado ${ }^{(1,2)}$.

Nesta pesquisa, por exemplo, durante a entrevista, as puérperas foram questionadas sobre eventos que pudessem desencadear estresse, tais como problema conjugal, falta de apoio familiar, conflitos familiares, violência, problemas financeiros e/ou preocupação com o curso da gestação. Fato é que a associação entre estresse e prematuridade obteve diferenças estatisticamente significantes nos grupos avaliados, pois mais de $70 \%$ das puérperas do grupo A relataram um ou mais eventos estressantes na gestação $(p=0,027)$. Pesquisa realizada em São Paulo com 865 mulheres também mostrou significância estatística ao relacionar o estresse materno pré-natal com a prematuridade ${ }^{(19)}$.Contudo, o resultado deste estudo nesse item deve ser interpretado com cautela, pois uma limitação desta pesquisa foi o fato de não ter utilizado nenhum instrumento acurado para detectar estresse nas puérperas.

Em uma análise mais minuciosa dessa associação, os autores destacam que os níveis dos hormônios adrenocorticotrófico (ACTH), beta-endorfina e cortisol, relacionados com a resposta humana em situações de estresse, se elevam no plasma sanguíneo de gestantes estressadas como resposta do seu sistema nervoso autônomo. Ao transpor a barreira placentária, em virtude do aumento dessas substâncias na concentração sérica, parece haver uma modificação na bioquímica do ambiente intrauterino que favorece o parto pré-termo ${ }^{(20)}$.

Dentre as variáveis clínicas, foi possível identificar diferenças com significância estatística nos grupos em primiparidade $(\mathrm{OR}=2,15)$, assistência pré-natal ausente ou inadequada $(\mathrm{OR}=5,06)$ e inter- corrências clínicas durante a gestação $(\mathrm{OR}=4,74)$. Dessa forma, segundo os dados encontrados, ser primípara, possuir assistência pré-natal deficitária e apresentar intercorrências clínicas gestacionais aumentam as chances de prematuridade em duas, cinco e quatro vezes, respectivamente. (Tabela 2).

No que concerne à primiparidade, foram encontradas publicações com resultados similares e diferentes ao desta pesquisa ${ }^{(19,17}$. Segundo esses estudos consultados, a paridade é um fator que, em conjunto com fatores sociodemográficos, tais como idade e baixa escolaridade, pode elevar consideravelmente o risco de um nascimento prematuro ${ }^{(2,3)}$.

Praticamente $60 \%$ das puérperas de prematuros realizaram menos de cinco consultas de pré-natal e apresentaram cinco vezes mais chances de prematuridade em relação às mães de crianças a termo, isso corrobora outros autores consultados neste assunto ${ }^{(13,17)}$. É importante destacar que mães de recém-nascidos prematuros têm a gestação interrompida, logo, naturalmente, realizaram menos consultas pré-natais. Por outro lado, considerando que em uma gravidez normal o retorno deve ocorrer a cada quatro semanas, e que mais de $70 \%$ das mães desta iniciaram o pré-natal com até 12 semanas, foi considerado adequado apenas o pré-natal das mães que contavam com, no mínimo, 5 consultas ao completar 28 semanas de gestação.

Não foi possível identificar diferenças estatisticamente significantes na relação entre etilismo e tabagismo com a prematuridade, até mesmo porque mais de $90 \%$ das entrevistadas de ambos os grupos negaram tais hábitos durante a gestação. Esse resultado é similar aos de outros estudos brasileiros analisados $^{(2,3)}$.

Neste estudo,o percentual de puérperas com IMC alterado foi semelhante em ambos os grupos, portanto, não houve diferenças estatisticamente significantes nesta associação entre os grupos. Mesmo assim, é fato bem estabelecido na literatura que a nutrição inadequada é um fator de risco para nascimentos prematuros e está intimamente relacionada com o baixo nível socioeconômico das gestantes ${ }^{(13)}$.

O aborto foi outra variável investigada em que não se obteve diferença estatisticamente significante entre os grupos analisados $(\mathrm{p}=0,495)$. Tal achado também foi identificado em uma investigação anterior consultada $\mathrm{a}^{(9)}$. O fato de possuir histórico obstétrico de nascimento prematuro anterior também não atuou como fator de risco para prematuridade neste estudo $(\mathrm{p}=0,106)$, o que talvez possa ser 
explicado pelo número reduzido de multíparas na amostra. Por sua vez, autores encontrados afirmam que após um parto prematuro espontâneo, o risco de repetição varia de 14 a $22 \%$, podendo ultrapassar $60 \%$ após três partos prematuros consecutivos ${ }^{(12)}$.

Parte substancial da amostra analisada do grupo A (84\%) apresentou intercorrências clínicas durante a gestação $(p<0,001)$, possivelmente, em decorrência do número de chances aumentado em mais de quatro vezes para estes fenômenos $(\mathrm{OR}=$ 4,74 ). Pelos resultados encontrados nesta pesquisa, foi possível identificar que as principais intercorrências clínicas durante a atual gestação das mães de prematuros foram: rotura prematura de membranas (19\%), DHEG (14\%), sangramento vaginal (14\%) e associação entre DHEG e ITU (12\%). Vale salientar ainda que $16 \%$ da amostra pesquisada não apresentaram nenhuma intercorrência clínica durante a gestação. Por outro lado, no grupo B, foi possível verificar que a principal intercorrência foi ITU isoladamente, ou seja, não associada com outras complicações clínicas. Outro dado relevante é que $47 \%$ das puérperas que tiveram recém-nascidos a termo não apresentaram intercorrências clínicas durante a gestação.

Ao associar cada uma dessas intercorrências com a prematuridade foi possível constatar significância estatística com a DHEG $(\mathrm{p}<0,001)$ e como sangramento vaginal $(\mathrm{p}<0,001)$, corroborando outros artigos encontrados nesta temática ${ }^{(9,15)}$. De todas as patologias que se manifestam na gravidez, a hipertensão é a mais frequente. A pré-eclampsia e a eclampsia são as duas formas clínicas principais da DHEG. Como sua etiologia ainda é desconhecida e a sua cura só ocorre após a expulsão da placenta, é importante que os profissionais de saúde estejam familiarizados com os seus sinais e sintomas (hipertensão, edema e proteinúria), a fim de detectar precocemente casos desse distúrbio. $\mathrm{O}$ prolongamento da gestação pelo maior tempo possível pode ser uma alternativa a fim de que o feto possa alcançar maturidade, mas a indução do parto deve acontecer quando há risco fetal ${ }^{(1)}$.

A ruptura prematura de membrana (RPM) e a ITU também ocorreram com frequência no grupo $\mathrm{A}$, porém, no grupo $\mathrm{B}$ a frequência dessas intercorrências também obteve um resultado considerável. Portanto, na amostra desta pesquisa não houve associação estatisticamente significante entre RPM e ITU com o nascimento de prematuros. Por sua vez, o sangramento vaginal ocorreu isoladamente em $14 \%$ do grupo A, com evidência estatisticamente significante $(\mathrm{p}<0,001)$; e em $13 \%$ dos quando associado a outras intercorrências clínicas nas gestantes com parto prematuro. Outros autores já relacionaram o sangramento vaginal com o parto prematuro, confirmando os resultados desta pesquisa $^{(9,15)}$. É importante ressaltar que neste estudo

Tabela 2 - Distribuição das variáveis clínicas das mães de crianças prematuras e a termo. Imperatriz, MA, 2011.

\begin{tabular}{|c|c|c|c|c|c|c|c|}
\hline & \multicolumn{2}{|c|}{ Prematuros } & \multicolumn{4}{|c|}{ Não prematuros } & \\
\hline VARIÁVEIS & $\mathrm{N}$ & $\%$ & $\mathrm{~N}$ & $\%$ & OR & IC & $\mathrm{p}$ \\
\hline
\end{tabular}

Tabagismo

\begin{tabular}{llllllll} 
Sim & 4 & $7 \%$ & 5 & $9 \%$ & 0,78 & $0,19-3,08$ & 0,721 \\
Não & 54 & $93 \%$ & 53 & $91 \%$ & & & \\
Etilismo & & & & & & & \\
Sim & 5 & $9 \%$ & 5 & $9 \%$ & 1,00 & $0,27-3,65$ & 1,002 \\
Não & 53 & $91 \%$ & 53 & $91 \%$ & & & \\
IMC & & & & & & & \\
Eutrófico & 33 & $60 \%$ & 36 & $65 \%$ & & & \\
Alterado & 22 & $40 \%$ & 19 & $35 \%$ & 0,79 & $0,36-1,71$ & 0,553 \\
\hline
\end{tabular}




\begin{tabular}{lllllllll}
\hline \multicolumn{8}{c}{ Prematuros } & \multicolumn{2}{l}{ Não prematuros } \\
\hline VARIÁVEIS & $\mathrm{N}$ & $\%$ & $\mathrm{~N}$ & $\%$ & OR & IC & $\mathrm{p}$ \\
\hline
\end{tabular}

Paridade

Primípara

35

Multípara

Aborto

Sim

11

47

$19 \%$

14

$81 \%$

44

$24 \%$

0,73

0,30- 1,79

0,495

Não

5

$9 \% \quad 1$

$2 \%$

5,37

$0,60-47,5$

0,106

Não

$91 \%$

57

$98 \%$

Pré-natal

Não houve ou $<5$ consultas

33

$57 \%$

12

$21 \%$

5,06

$2,22-11,49$

$<0,001 *$

$\geq 5$ consultas

$43 \% \quad 46$

$79 \%$

$1^{\text {a }}$ Consulta

$1^{\mathrm{o}}$ trim.

40

$73 \%$

45

$82 \%$

$0,59 \quad 0,23-1,46$

0,257

$2^{\circ}$ e $3^{\circ}$ trim.

$27 \%$

10

$18 \%$

Intercorrências

\begin{tabular}{llllllll} 
Sim & 49 & $84 \%$ & 31 & $53 \%$ & 4,74 & $1,97-11,411$ & $<0,001 *$ \\
Não & 9 & $16 \%$ & 27 & $47 \%$ & & & \\
\hline
\end{tabular}

OR-Odds ratio (Razão de chance)

*valor p significativo

IC - Intervalo de Confiança de 95\%

foi considerado o sangramento vaginal ocorrido em qualquer momento antes de 37 semanas de gestação. De acordo com dados encontrados na literatura, na primeira metade da gestação esses sangramentos normalmente estão associados à ameaça de abortamento, enquanto na segunda estão relacionados com a placenta prévia ou com o descolamento prematuro de placenta $^{(1,11)}$.

\section{CONSIDERAÇÕES FINAIS}

Com base nos resultados deste estudo, pode-se concluir que na amostra analisada as variáveis que tiveram associação estatisticamente significante com a prematuridade foram: renda mensal inferior a 2 SM; estresse na gestação; primiparidade; assistência pré-natal ausente ou inadequada; e intercorrências clínicas na gestação (DHEG e sangramentos vaginais).Apesar disso, esta investigação possui limitações tais como possuir uma amostra pequena, conveniente, oriunda de um único serviço, com alguns dados baseados no relato materno e registros clínicos como no caso do estresse do IMC, respectivamente. Dessa forma, a generalização dos seus resultados em relação à população geral fica prejudicada.

Na região onde se desenvolveu esta pesquisa, denominada Bico do Papagaio, responsável pela assistência perinatal do Sul do Maranhão e cidades adjacentes do Tocantins e Pará, não há ainda dados publicados a respeito deste tema. Portanto, mesmo com as limitações metodológicas supracitadas, esta pesquisa foi importante para que os profissionais de saúde locais e das adjacências conheçam os 
principais fatores de risco para prematuridade que acometem essa região.

A prematuridade é um problema de saúde pública complexo devido à inter-relação existente entre os diversos fatores de risco desencadeantes desse evento. Assim, principalmente em partos prematuros espontâneos, não se pode trabalhar com a hipótese de que apenas um fator foi o responsável pelo nascimento prematuro, mas sim considerá-lo um fato com múltiplas causas que podem ser independentes ou interdependentes. Dessa forma, novos estudos em outras regiões do Brasil, com amostras mais significativas e com melhor delineamento metodológico, são necessários para ampliar a discussão e trazer novos esclarecimentos sobre este tema.

\section{REFERÊNCIAS}

1 Montenegro RF. Obstetrícia fundamental. $11^{\mathrm{a}} \mathrm{ed}$. Rio de Janeiro: Guanabara Koogan; 2008.

2 Cascaes AM, Gauche H, Baramarchi FM, Borges CM, Perez KG. Prematuridade e fatores associados no Estado de Santa Catarina, Brasil, no ano de 2005: análise dos dados do Sistema de Informações sobre Nascidos Vivos. Cad Saúde Pública. 2008; 24(5): 1024-32.

3 Salge AKM, Viera AVC, Aguiar AKA, Lobo SF, Xavier RM, Zatta LT, et al.Fatores maternos e neonatais associados à prematuridade. Rev Eletr Enferm. 2009; 11(3): 642-6.

4. Blencowe H, Cousens S, Oestergaard MZ, Chou D, Moller AB, Narwal R, et al. Nacido demasiado pronto: informe de acción global sobre nacimientos prematuros [Internet]. Genebra: OMS; [2012] [citado 2012 maio 27]. Disponível em: www.who.int/ $\mathrm{pmnch} / \mathrm{media} /$ news/2012/preterm_birth_report/ es/index.html

5 Martins MG, Santos GHN, Sousa MS, Costa JEFB, Simões VMF. Associação da gravidez na adolescência e prematuridade. Rev Bras Ginecol Obstet. 2011; 33(11): 354-360.

6 Almeida MVL. Prematuridade. In: Chaves Netto H, Moreira de Sá, RA. Obstetrícia básica. $2^{\text {a }}$ ed. São Paulo: Atheneu; 2007.

7 Silveira MF, Santos IS, Matijasevich A, Malta DC, Duarte EC. Nascimentos pré-termo no Brasil entre 1994 e 2005 conforme o Sistema de Informações sobre Nascidos Vivos (SINASC). Cad Saúde Pública 2009; 25(6): 1267-75.

8 Silveira MF, Victora CG, Barros AJD, Santos IS, Matijasevich A, Barros A, et al. Determinantes de nascimento pré-termo na coorte de nascimentos de 2004, Pelotas, Rio Grande do Sul, Brasil. Cad Saúde Pública 2010; 26(1): 185-94.

9 Silva AMR, Almeida MF, Matsuo T, Soares DA. Fatores de risco para nascimentos pré-termo em Londrina, Paraná, Brasil. Cad Saúde Pública. 2009; 25(10): 2125-38.

10 Ramos HAC, Cuman RKN. Fatores de risco para prematuridade: pesquisa documental. Esc Anna Nery. 2009; 13 (2): 297-304.

11 Graciete H, Santos N, Martins MG, Sousa MS. Gravidez na adolescência e fatores associados com baixo peso ao nascer. Rev Bras Ginecol Obstet. 2008; 30(5): 224-31.

12 Bittar RE, Zugaib M. Indicadores de risco para o parto prematuro. Rev Bras Ginecol Obstet. 2009; 31(4): 203-9.

13 UchimuraTT, Pelissari, Uchimura NS. Baixo peso ao nascer e fatores associados. Rev Gaúcha Enferm 2008; 29(1): 33-8.

14 Musilová I, Kacerovský M, Tambor V, Tosner J. Proteomics and biomarkers for detection of preterm labor: a systematic review. Ceska Gynekol. 2011; 76(1): 37-45.

15 Silva LA, Silva RGA, Rojas PFB, Laus FF, Sakae TM. Fatores de risco associados ao parto pré-termo em hospital de referência de Santa Catarina. Rev AMRIGS [Internet] 2009 [citado 2011 maio 18]; 53 (4): 354-60. Disponível em: www.amrigs.org.br/ revista/53-04/08-445_fatores_de_risco.pdf.

16 Pattel RR, Steer P, Doyle P, Little MP, Elliott P. Does gestation vary by ethnic group? - a London-based study of over 122.000 pregnancies with spontaneous onset of labour. Int J Epidemiol. 2004; 33(1): 107-13.

17 Ferraz RT, Neves ET. Fatores de risco para baixo peso ao nascer em maternidades públicas. Rev Gaúcha Enferm 2011; 32(1): 86-92.

18 Maia RRP, Souza JMP. Fatores associados ao baixo peso ao nascer em município do norte do Brasil. Rev Bras Crescimento Desenvolv Hum. 2010; 20(3): 735-44. 
19 Rondó PH, Ferreira RF, Nogueira F, Ribeiro MC, Lobert H, Artes R. Maternal psychologicalanddistress as predictor of low birth weight, prematurity and intra uterine growth retardation. Eur J ClinNutr. 2003; 57(2): 266-72.
20 Latendresse G, Ruiz RJ. Maternal corticotropin-releasing hormone and the use of selective serotonin reuptake inhibitors independently predict the occurrence of preterm birth. J Midwifery Womens Health. 2011; 56(2): 118-26.

Endereço do autor / Dirección del autor / Author's address:

Márcio Flávio Moura de Araújo

Rua Amazonas, 1359, Centro

65901-520, Imperatriz, MA

E-mail:marciofma@yahoo.com.br 